\title{
Extracellular volatilomic alterations induced by hypoxia in breast cancer cells
}

\author{
Ravindra Taware ${ }^{1} \cdot$ Khushman Taunk $^{1} \cdot$ Totakura V. S. Kumar $^{2}$ • Jorge A. M. Pereira ${ }^{3}$. José S. Câmara ${ }^{3,4}$. \\ H. A. Nagarajaram ${ }^{5}$. Gopal C. Kundu ${ }^{2,6} \cdot$ Srikanth Rapole ${ }^{1}[1]$
}

Received: 5 September 2019 / Accepted: 3 January 2020 / Published online: 24 January 2020

○) Springer Science+Business Media, LLC, part of Springer Nature 2020

\begin{abstract}
Introduction The metabolic shift induced by hypoxia in cancer cells has not been explored at volatilomic level so far. The volatile organic metabolites (VOMs) constitute an important part of the metabolome and their investigation could provide us crucial aspects of hypoxia driven metabolic reconfiguration in cancer cells.

Objective To identify the altered volatilomic response induced by hypoxia in metastatic/aggressive breast cancer (BC) cells. Methods BC cells were cultured under normoxic and hypoxic conditions and VOMs were extracted using HS-SPME approach and profiled by standard GC-MS system. Univariate and multivariate statistical approaches $\left(p<0.05, \log _{2}\right.$ $\mathrm{FC} \geq 0.58 / \leq-0.58, \mathrm{PC} 1>0.13 /<-0.13$ ) were applied to select the VOMs differentially altered after hypoxic treatment. Metabolic pathway analysis was also carried out in order to identify altered metabolic pathways induced by the hypoxia in the selected BC cells.

Results Overall, 20 VOMs were found to be significantly altered $(p<0.05, \mathrm{PC} 1>0.13 /<-0.13)$ upon hypoxic exposure to $\mathrm{BC}$ cells. Further, cell line specific volatilomic alterations were extracted by comparative metabolic analysis of aggressive (MDA-MB-231) vs. non-aggressive (MCF-7) cells incubated under hypoxia and normoxia. In this case, 15 and 12 VOMs each were found to be significantly altered in aggressive cells when exposed to hypoxic and normoxic condition respectively. Out of these, 9 VOMs were found to be uniquely associated with hypoxia, 6 were specific to normoxia and 6 were found common to both the conditions. Formic acid was identified as the most prominent molecule with higher abundance levels in aggressive as compared to non-aggressive cells in both conditions. Furthermore, metabolic pathway analyses revealed that fatty acid biosynthesis and nicotinate and nicotinamide metabolism were significantly altered in aggressive as compared to non-aggressive cells in normoxia and hypoxia respectively.

Conclusions Higher formate overflow was observed in aggressive cells compared to non-aggressive cells incubated under both the conditions, reinforcing its correlation with aggressive and invasive cancer type. Moreover, under hypoxia, aggressive cells preferred to be bioenergetically more efficient whereas, under normoxia, fatty acid biosynthesis was favoured when compared to non-aggressive cells.
\end{abstract}

Electronic supplementary material The online version of this article (https://doi.org/10.1007/s11306-020-1635-x) contains supplementary material, which is available to authorized users.

Srikanth Rapole

rsrikanth@nccs.res.in

1 Proteomics Lab, National Centre for Cell Science, Ganeshkhind, Pune 411007, India

2 Laboratory of Tumor Biology, Angiogenesis and Nanomedicine Research, National Centre for Cell Science (NCCS), Ganeshkhind, Pune 411007, India

3 CQM - Centro de Química da Madeira, Universidade da Madeira, Campus Universitário da Penteada, 9020105 Funchal, Portugal
4 Faculdade de Ciências Exatas E da Engenharia, Universidade da Madeira, Campus Universitário da Penteada, 9020-105 Funchal, Portugal

5 Department of Systems and Computational Biology, School of Life Sciences, University of Hyderabad, Hyderabad 500046, India

6 Present Address: School of Biotechnology, KIIT Deemed to be University, Institute of Eminence, Bhubaneswar 751024, India 


\section{Graphic Abstract}

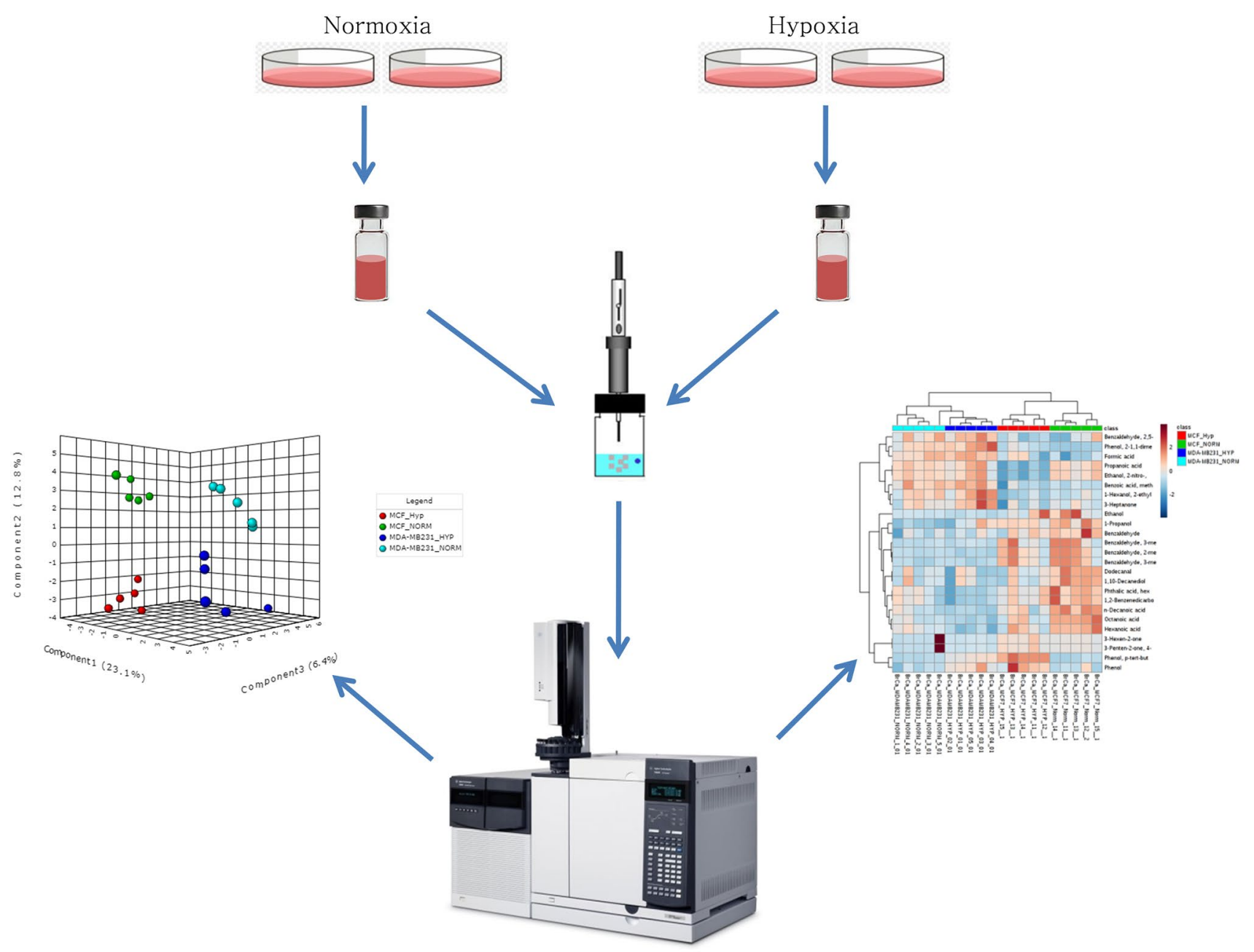

Keywords Hypoxia $\cdot$ Volatile organic metabolites (VOMs) $\cdot$ GC-MS $\cdot$ Volatilomics $\cdot$ Breast cancer

\section{Introduction}

Worldwide, breast cancer (BC) is the most prominent malignant condition observed in women, with an overall incidence rate only second to lung cancer and the fifth in terms of mortality. Approximately 2.1 million new cases were diagnosed in 2018, accounting for $11.6 \%$ of total global cancer burden (Bray et al. 2018). Although, recent trends of early diagnosis and improved chemotherapeutic interventions have led to enhanced overall disease free survival rate but still, approximately $30 \%$ of the localized BC cases detected at initial stages eventually progress into distant organ metastases (O'Shaughnessy 2005).

Higher cell proliferation rate and increased oxygen consumption in solid tumor lead to the development of intratumoral hypoxic environment (Harris 2002; Vaupel et al.
2007). It is well established phenomenon that hypoxic microenvironment is a major driver of the cancer progression, invasiveness and metastasis (Chaturvedi et al. 2013; Semenza 2016; Zhang et al. 2016). Hypoxic condition induces several adaptive responses in the cancer cells which allow them to adjust to hostile environment (Vaupel 2004). These effects are carried out by the group of transcription factors known as hypoxia inducible factors (HIF), which comprise of the $\alpha$ and $\beta$ heterodimers viz. HIF- $1 \alpha$, HIF- $2 \alpha$ and HIF-3 $\alpha$ (Prabhakar and Semenza 2012; Semenza 2012). In particular, HIF1 $\alpha$ is considered as a master regulator of the hypoxia-driven responses that are critical for the malignant features of the tumor (Semenza 2010a, b).

Hypoxia induced stabilization of HIF1 $\alpha$ drives an altered gene expression pattern in cancer cells which results in 
proteomic and metabolic adaptations better suited to cancer cells survival and proliferation in hostile microenvironment (Denko et al. 2003; Favaro et al. 2011; Jaakkola et al. 2001; Wang et al. 1995). The phenomenon such as 'Warburg effect' where cancer cells prefer aerobic glycolysis over mitochondrial oxidative phosphorylation to generate energy, even when adequate oxygen is available, is one of the most notable metabolic shifts observed in malignant condition (Warburg 1956; Warburg et al. 1927). It is now well established that HIF1 $\alpha$ once stabilized under hypoxic condition, drives Warburg effect by repressing mitochondrial oxidative phosphorylation and promoting aerobic glycolysis (Weidemann and Johnson 2008). Moreover, HIF1 $\alpha$ also upregulates several cancer specific isoforms of glycolytic enzymes including glucose transporter (Semenza 2010a, b). Furthermore, it is also reported that, HIF1 $\alpha$ control expression of hypoxia regulated miRNA (HRM) such as miR-210 by modifying the strength of hypoxia response elements (HREs) (Huang et al. 2009). Interestingly, it is now known that, miRNA-210 regulates the mitochondrial metabolic shift from oxidative phosphorylation to aerobic glycolysis by inhibiting electron transport chain complexes (Chen et al. 2010; Fasanaro et al. 2009).

Therefore, it is evident that, hypoxic microenvironment induces prominent metabolic changes in cancer cells to adapt, survive and proliferate in challenging surroundings. Consequently, several metabolomic studies have been attempted to identify metabolic patterns associated with the hypoxia-induced metabolic alterations. Such metabolic patterns would be useful to monitor disease progression and identify targets for better therapeutic management (Armitage et al. 2015; Intlekofer et al. 2015; Tsai et al. 2013; Yang et al. 2018). In this regard, an isotope-assisted metabolomics study by Metallo et al. reported that hypoxic cancer cells undergo reductive glutaminolysis for de novo lipid synthesis carried out by IDH1 enzyme (Metallo et al. 2012). Furthermore, Mashimo et al. employed $\mathrm{C}^{13} \mathrm{NMR}$ and $\mathrm{C}^{13}$ labelled tracers and identified acetate as an important energy source for glioma (Mashimo et al. 2014). This observation was further supported by another study which revealed that acetate functions as an epigenetic modulator and activates lipogenic genes in cancer cells under hypoxic stress (Gao et al. 2016).

Though, metabolic investigation of cancer is a routinely followed tool to get meaningful insights of differential metabolomics response but unfortunately, volatile component of the metabolome is often ignored. Volatile organic metabolites (VOMs) comprise significant part of the metabolome which could complement and shed light on crucial aspects of metabolic dysregulations that occur during various pathophysiologies (Cavaco et al. 2018; Silva et al. 2011; Taunk et al. 2018; Taware et al. 2017, 2018).Therefore, in this study, we have carried out characterization of the VOMs produced by the MCF-7 (non-aggressive/non-metastatic) and MDA-MB-231( aggressive/metastatic)BC cell lines grown under normoxic and hypoxic conditions to identify the hypoxia driven specific volatilomic signature. Moreover, we also attempted to establish the hypoxia mediated volatilomic alterations as well as related metabolic pathways specific to the aggressive phenotype of breast cancer cells. To the best of our knowledge, this is the first attempt to define the hypoxia driven volatilomic signature of the breast cancer in a cell line model.

\section{Materials and methods}

\subsection{Cell culture and hypoxia treatment}

Breast cancer cell lines MCF-7 and MDA-MB-231 were acquired from American Type Culture Collection (ATCC, USA).The MCF-7 and MDA-MB-231 cells hereafter referred as non-aggressive and aggressive respectively. Cells were seeded at a density of $2 \times 10^{6}$ cells $/ \mathrm{mL}$ in high glucose containing Dulbecco's Modified Eagle Medium (GIBCO USA) supplemented with $10 \%$ foetal bovine serum (GIBCO, USA) along with penicillin (100 units) and $100 \mu \mathrm{g} / \mathrm{mL}$ streptomycin (Himedia, India). Cells were incubated under humidified condition at $37{ }^{\circ} \mathrm{C}$ and $5 \% \mathrm{CO}_{2}$ for 24 to $48 \mathrm{~h}$ or until $80 \%$ confluency was achieved. Cells were washed with serum free high glucose containing DMEM media to remove the residual traces of FBS. Both cell lines were then placed under normoxic condition $\left(37^{\circ} \mathrm{C}, 5 \% \mathrm{CO}_{2}, 74 \% \mathrm{~N}_{2}\right.$ and $\left.21 \% \mathrm{O}_{2}\right)$ or hypoxic condition $\left(37^{\circ} \mathrm{C}, 5 \% \mathrm{CO}_{2}, 94 \% \mathrm{~N}_{2}\right.$ and $1 \% \mathrm{O}_{2}$ ) for $48 \mathrm{~h}$ in serum-free high glucose containing DMEM medium. Moreover, same experiment was carried out without cells and considered as a negative control of the study. The hypoxia incubator chamber (StemCell Technologies, USA) was used for the hypoxic treatment.

\subsection{Sample processing and VOMs extraction}

The conditioned media of the normoxia or hypoxia treated cancer cells were collected and centrifuged at $300 \times \mathrm{g}$ for $10 \mathrm{~min}$ at $4{ }^{\circ} \mathrm{C}$, supernatant separated, labelled and stored at $-80{ }^{\circ} \mathrm{C}$ until further use. The VOMs from the conditioned media were extracted using a Headspace Solid-Phase Microextraction (HS-SPME) sampling approach as described elsewhere (Silva et al. 2011). Briefly, $4 \mathrm{~mL}$ of sample were acidified with $0.5 \mathrm{~mL}$ of $5 \mathrm{M}$ hydrochloric acid (Merck, Germany) and salting out effect was achieved by adding $0.8 \mathrm{~g}$ of sodium chloride (Merck, Germany).The sample was continuously agitated at $800 \mathrm{rpm}$ and incubated at $40{ }^{\circ} \mathrm{C}$ for $1 \mathrm{~h}$ with carboxen/polydimethylsiloxane SPME fibre (CAR/ PDMS, $75 \mu \mathrm{m}$, Supelco, USA) exposed into the headspace of the sampling vial. The negative control samples were processed in same manner as experimental samples. 


\subsection{Volatilomic data acquisition and pre-processing}

The VOMs adsorbed on the CAR/PDMS fibre were desorbed in the back inlet of the GC (Agilent 7890B, USA) for $6 \mathrm{~min}$ at $250{ }^{\circ} \mathrm{C}$. The VOMs were further separated using BP-20 (SGE, Germany) fused silica capillary column $(60 \mathrm{~m} \times 0.25 \mathrm{~mm} \times 0.25 \mu \mathrm{m})$ connected to quadrupole inert mass selective detector (Agilent 5977A, USA).The GC oven temperature programme and MS parameters are mentioned in supplementary information 2 . The $\mathrm{m} / \mathrm{z}$ range of 30 to 300 was scanned for the data acquisition and spectra were recorded at $70 \mathrm{eV}$. For each treatment, five biological replicates of both the cell lines were randomly acquired. Each biological replicate was acquired in technical duplicate. Pooled sample was prepared by adding equal quantity of all the samples together and acquired at regular interval of 5 runs which served as quality control measure (Supplementary Fig. 1). ChemStation data analysis software (Agilent, USA) was used to integrate the chromatograms. Metabolite identification was achieved by NIST11 mass spectral library linked with the Agilent ChemStation data analysis software using minimum match score of $\geq 75 \%$. VOMs showing missing values $>20 \%$ of the samples were removed from the analysis.

\subsection{Statistical and pathways analysis}

The data normalization and statistical analyses were carried out using the web based application Metaboanalyst 3.0 (Xia et al. 2009; Xia and Wishart 2011). The normalized data was subjected to univariate and multivariate statistical analysis. The univariate statistical analysis such as one-way ANOVA or Wilcoxon rank sum t-test $(p<0.05)$ and $\log _{2}$ fold change $(\geq 0.58 / \leq-0.58)$ were performed to evaluate the significant differences among the VOMs produced by non-aggressive and aggressive cells under hypoxia and normoxia. The unsupervised multivariate statistical classification model, namely the Principal Component Analysis (PCA) was used to visualise the distribution pattern of variables among hypoxic and normoxic groups. Important metabolites were selected based upon PCA loadings. To select the most important VOMs that discriminate between the two groups, combination of the univariate and multivariate statistical approach was used. Hierarchical cluster analysis (HCA) was performed to identify inherent clustering patterns associated with hypoxic and normoxic samples. Moreover, the volatilomic profiles of hypoxic and normoxic samples were used to get a global view of the altered metabolic pathways by using MetPA tool in Metaboanalyst 3.0 web application (Xia et al. 2011). Metabolomics standard initiative (MSI) guidelines were strictly followed during the analysis of volatilomics data
(Griffin et al. 2007). MSI compliant metadata for identified VOMs is supplied as supporting information 2.

\section{Results}

\subsection{Establishment of the hypoxia induced BC cell volatilomic signature}

HS-SPME coupled to GC-MS detected total 71 VOMs in the conditioned media of the non-aggressive and aggressive cells grown under normoxic and hypoxic conditions. Total 47 VOMs found to be endogenous in nature with criteria of either exclusively found in the culture media with cells or average abundance fold change $>3$ as compared to negative control (Supplementary Table 1). Univariate and multivariate statistical approaches were used for the identification of volatilomic signature associated with the hypoxia. Data normalization was carried out to remove the bulk differences from the data matrix and to make variables more comparable. The combination of median normalization, cube root transformation and auto scaling yielded Gaussian distribution of the data. Furthermore, data thus normalized were subjected to univariate statistical analysis using oneway Analysis of Variance (ANOVA) to select the metabolites showing statistically significant differential abundances. A total of 26 metabolic features were identified by the ANOVA with the threshold of false discovery rate corrected $p<0.05$ (Supplementary Table 2). Moreover, multivariate statistical analysis such as PCA was also carried out in order to visualise the pattern associated with the hypoxia-induced metabolic changes (Fig. 1).The PCA score plots depict the separation of the four groups belonging to non-aggressive and aggressive cells grown under normoxia and hypoxia (Fig. 1a and $b$ ).The segregation of the groups in PCA model indicate that metabolic differences are dependent of the cell line and growing conditions. These findings were further complemented by Hierarchical Cluster Analysis (HCA) which indicates the robust separation of all groups based upon metabolic concentration variation owing to difference in cell type and treatment (Fig. 1c). Combination of univariate and multivariate approaches (FDR $<0.05, \mathrm{PC} 1>0.13 /<-0.13$ ) identified 20 VOMs significantly altered upon hypoxic exposure in both the cell lines (Table 1).

\subsection{Comparative analysis of hypoxia-induced metabolic reconfiguration in aggressive and non-aggressive BC cells}

To understand if hypoxia induces different metabolic responses in aggressive with respect to non-aggressive cells, we carried out a comparative volatilomic analysis of both cell types grown under hypoxic and normoxic 
$\mathbf{a}$

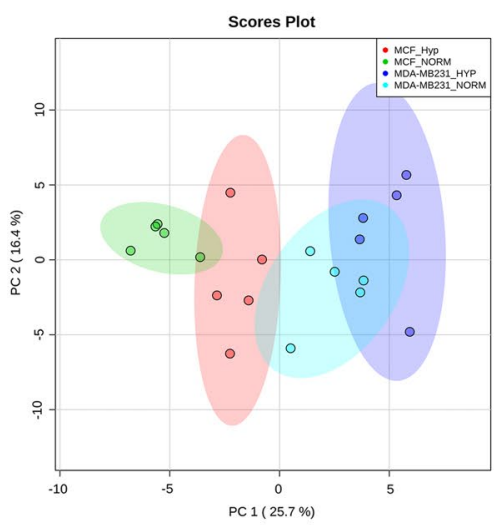

b

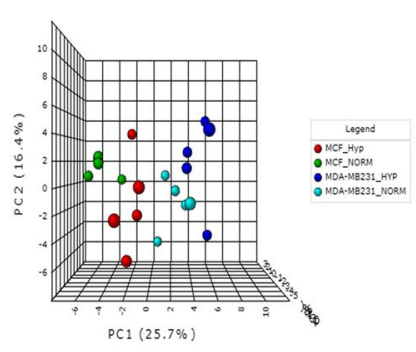

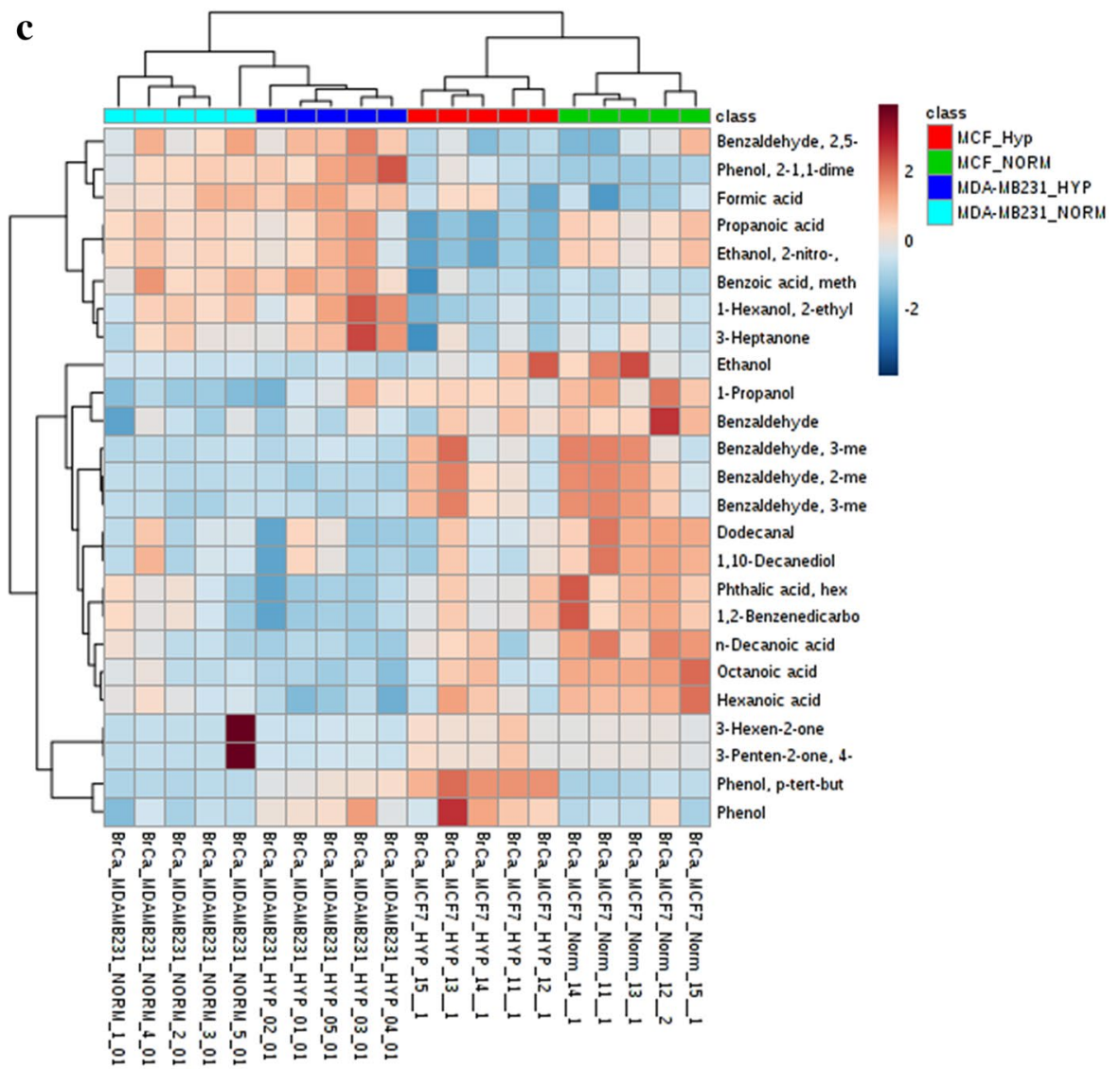

Fig. 1 Multivariate statistical analysis of the hypoxia-inducible volatilome of BC cells. a PCA 2D score plot and b PCA 3D score plot of the VOMs produced by non-aggressive and aggressive cells under normoxic and hypoxic conditions and $\mathbf{c}$ hierarchical cluster analysis (HCA) of VOMs produced by non-aggressive and aggressive cells

conditions. Combination of univariate and multivariate statistical approaches $\left(\mathrm{PC} 1>0.13 /<-0.13, p<0.05\right.$ and $\log _{2}$ $\mathrm{FC} \geq 0.58 / \leq-0.58$ ) were used to select the $15 \mathrm{VOMs}$ with statistically significant differential abundance in aggressive cells as compared to non-aggressive cells in hypoxic condition (Supplementary Fig. 2A). Robust segregation was observed in PCA as well as HCA plot which indicate differential metabolic response of the cells to the hypoxic condition (Fig. 2). To make sure that the VOMs selected are hypoxia-specific and differentially altered, comparative volatilomic analysis between aggressive and non-aggressive cells under normoxic condition was also carried out. Vigorous separation was observed in PCA score plots indicating differential volatilomic response to normoxic condition in aggressive phenotype of the BC cells (Fig. 3, and Supplementary Fig. 2B). Finally, the discriminatory VOMs identified for hypoxic $(n=15)$ and normoxic conditions $(n=12)$ were compared with each other using Venn diagram analysis (Venny 2.0). Nine unique VOMs for hypoxic, six specific for normoxic condition as well as six common metabolites grown under normoxic and hypoxic conditions represented as heatmap. Clustering was performed using 'complete' algorithm and distance was measured by correlation. Complete algorithm used for the clustering and distance measured by correlation calculations

to both conditions were identified (Fig. 4, Supplementary Fig. 3).

\subsection{Hypoxia-induced metabolic pathway analysis}

MetPa tool of the MetaboAnalyst web application was used to decipher the metabolic pathway alterations in the aggressive cells compared to non-aggressive cells in hypoxic as well as normoxic conditions. The pathway topography analysis showed in Fig. 5 reveals that under normoxia, the fatty acid biosynthesis is the most significantly altered pathway, while under hypoxia the nicotinate and nicotinamide metabolism was most affected. In contrast, the metabolic routes of methane, glyoxylate and dicarboxylate and pyruvate are relatively conserved among the conditions assayed. The differentially altered metabolic pathways are summarised in the Supplementary Table 3 . 
Table 1 Significant metabolic features altered upon hypoxic exposure in BC cells. The metabolites were selected by PCA loading score $>0.13 /<-0.13$, p-value $<0.05$ and $\mathrm{FDR}<0.05$

\begin{tabular}{llc}
\hline Sr. No & Compounds & PCA loading score \\
\hline 1 & 2-1,1-Dimethylethyl-phenol & 0.218 \\
2 & Formic acid & 0.199 \\
3 & Methyl benzoate & 0.180 \\
4 & 2-Ethyl-1-hexanol & 0.172 \\
5 & 2,5-Dimethyl-benzaldehyde & 0.163 \\
6 & 3-Heptanone & 0.139 \\
7 & 4,6-Dimethyl-2 heptanone & 0.136 \\
8 & 1-Propanol & -0.144 \\
9 & Benzaldehyde & -0.145 \\
10 & n-Propylacetate & -0.148 \\
11 & Ethanol & -0.159 \\
12 & 1,10-Decanediol & -0.169 \\
13 & Dodecanal & -0.178 \\
14 & Hexanoic acid & -0.182 \\
15 & 3-Methyl- benzaldehyde & -0.193 \\
16 & 2-Methyl- benzaldehyde & -0.197 \\
17 & Octanoic acid & -0.200 \\
18 & Hex-3-yl isobutyl ester phthalic acid & -0.205 \\
19 & 1,2-Benzenedicarboxylic acid, bis2- & -0.205 \\
& methylpropyl ester & -0.214 \\
\hline
\end{tabular}

\section{Discussion}

The reduced oxygen tension in the tumor microenvironment results in the activation of HIF transcription factors. In turn, HIFs drive the expression of numerous genes involved in adaptation of cancer cells to challenging surroundings. It is known that hypoxia influences the metabolic adaptation in cancer cells and several studies have already been carried out to identify the metabolic dysregulation that occurs during the hypoxic condition (Armitage et al. 2015; Intlekofer et al. 2015; Tsai et al. 2013; Yang et al. 2018). In this study, for the first time, we have carried out a comprehensive volatilomic analysis of non-aggressive and aggressive $\mathrm{BC}$ cells under hypoxic and normoxic microenvironments to understand if hypoxia induces any significant volatilomic alterations which could then be correlated with the aggressiveness of $\mathrm{BC}$.

First objective of the study was to check if hypoxia induces any significant volatilomic changes in the breast cancer cells. Multivariate statistical analysis (PCA) of both cell lines in hypoxic as well as normoxic conditions revealed distinct group segregation in score plot. This result indicated that hypoxia induces cell-specific volatilomic alterations. Accordingly, $20 \mathrm{VOMs}$ including alcohols, aldehydes, organic acids and ketones were found significantly altered in both $\mathrm{BC}$ cell types after hypoxic treatment. Further, to identify the aggressive cell specific volatilomic alterations, comparative univariate and multivariate statistical analyses between both the cell lines were undertaken, which revealed 15 differentially abundant VOMs in hypoxic as well as 12 differentially altered in normoxic condition. When these metabolite panels were compared to each other, we observed that 9 VOMs were specific to hypoxia, 6 were unique to normoxia and 6 were common to both conditions. Furthermore, the abundance of these common VOMs, either up or down, was conserved among the conditions assayed.

Formic acid was identified as a common metabolite which was found at elevated levels in aggressive as compared to non-aggressive cells in hypoxic as well as normoxic condition. It's recently known that serine to formate catabolism is up regulated in metastatic breast cancer animal model (Meiser et al. 2018). In same study, they also reported that increased production of formate in tumor is correlated with higher formate concentration in the plasma. Furthermore, it was also reported that increased formate overflow promotes cancer cell invasion in glioblastoma in a concentration dependent manner. Knockdown of MTHFD1L, the mitochondrial gene responsible for the synthesis of formate, leads to significant reduction of the invasion potential of glioblastoma. This phenotype was further rescued by the exogenous supply of formate to glioblastoma cells. These observations support the correlation between formate overflow and aggressive phenotype of the cancer.

Medium chain fatty acids such as octanoic and hexanoic acids were found to be reduced in aggressive cells when compared to non-aggressive cells in normoxic as well as hypoxic condition. In addition, another medium chain fatty acid n-decanoic acid was also found at lower levels in aggressive cells under normoxic condition. Overall, medium chain fatty acids showed reduced expression in aggressive cells under normoxic condition. These results specify that, medium chain fatty acids were getting consumed at higher rate in aggressive cells indicating excessively active fatty acid biosynthesis pathway under normoxic condition. On the other hand, propanoic acid, a short chain fatty acid level was elevated during hypoxic condition in aggressive cells. It is reported to be involved in immune-regulatory and inflammatory processes but, its role in malignant disease pathophysiology is not well scrutinized (Al-Lahham et al. 2010).

Ketonic metabolites such as 4-methyl-3-penten-2one, and 3-hexen-2-one were found at reduced levels in the aggressive cells, indicating their higher consumption as compared to non-aggressive cells under hypoxia. It is not surprising to find the lower levels of ketones, as these molecules are considered as preferred choice for energy generation due to their higher energy yield compared to other mitochondrial substrates under hypoxic condition 
$\mathbf{a}$

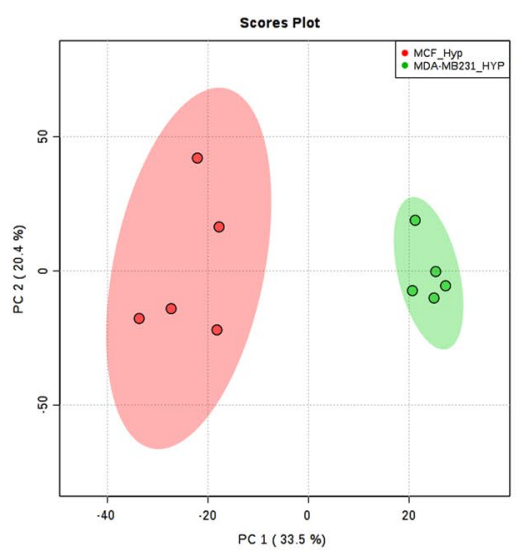

b

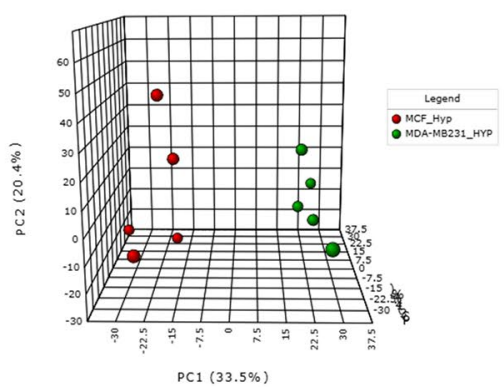

c

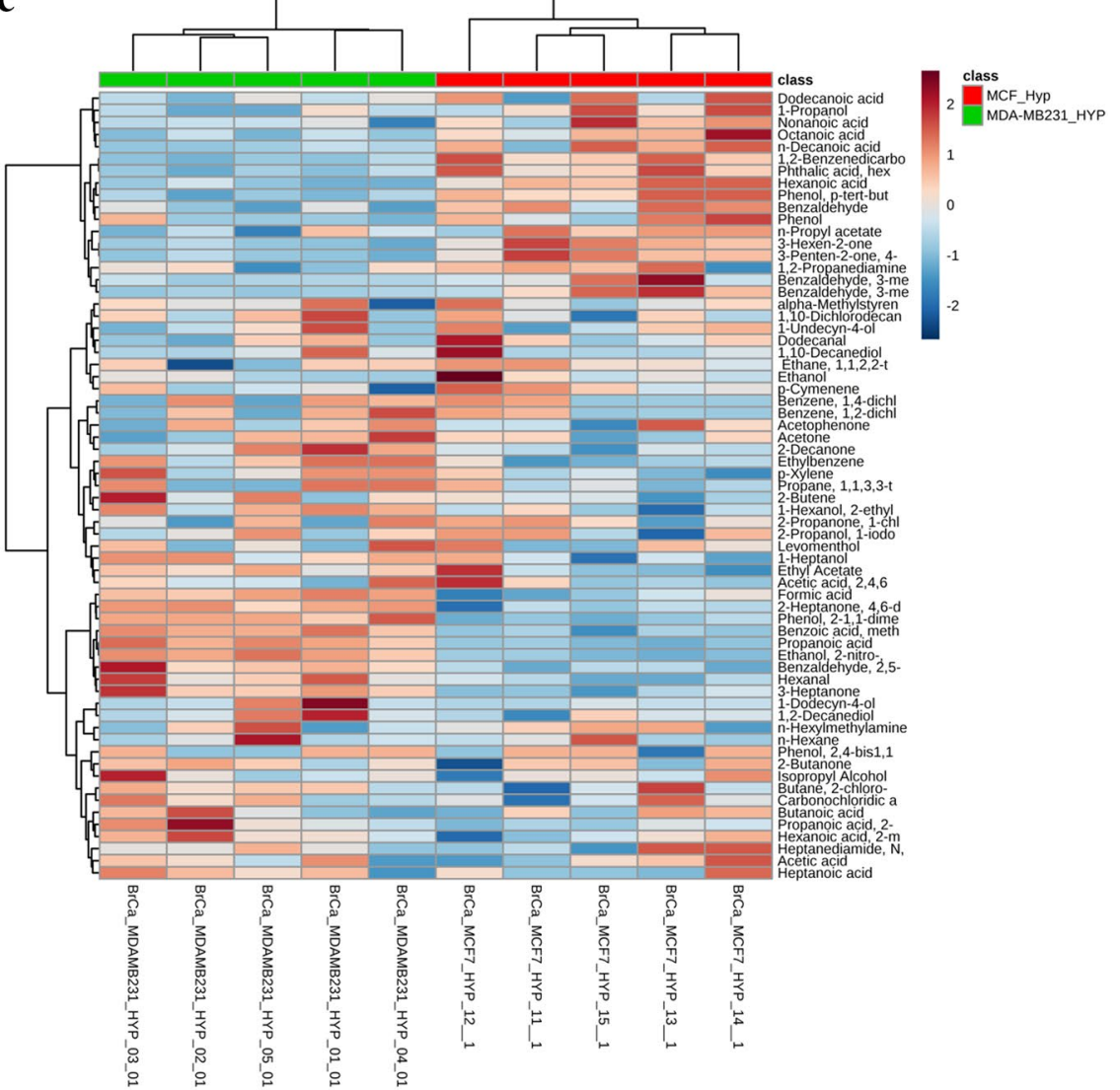

Fig. 2 a PCA 2D score plot and b PCA 3D score plot of the VOMs secreted by non-aggressive and aggressive cells under hypoxic condition. c Hierarchical cluster analysis (HCA) of the VOMS secreted by

(Martinez-Outschoorn et al. 2012). These findings indicate that aggressive cells are more efficient than non-aggressive cells in generating energy under hypoxic microenvironment. Moreover, several metabolic pathways were found to be altered in aggressive cells as compared to non-aggressive cells under hypoxic as well as normoxic condition. The most obvious find refers to nicotinate and nicotinamide metabolism which suggests that mitigation of hypoxic stress by aggressive cells involves bioenergetics adjustments. In contrast, under normoxia, fatty acid biosynthesis and glycerolipid metabolism were significantly altered indicating the enhanced predisposition of aggressive cells towards membrane synthesis and cell proliferation as compared to non-aggressive cells.

Taken together, results obtained from the volatilomic analysis of the $\mathrm{BC}$ cells differing in their aggressiveness under hypoxia as well as normoxia, revealed unique volatilomic signature. Further biochemical investigations into the differential volatilomic response could help to strengthen these observations. Moreover, additional follow up studies could be undertaken with other malignant non-aggressive and aggressive cells grown under hypoxic condition represented as heatmap. Euclidean algorithm was used for the clustering and distance was measured by Ward Distance calculations

cell lines in order to identify the unique as well as common volatilomic signature associated with different types of cancers.

\section{Conclusions}

Hypoxia induces distinct volatilomic responses in BC cells which can be efficiently extracted, analyzed and identified by HS-SPME coupled to GC-MS. A panel of 20 statistically significant VOMs were differentially altered in BC cells upon hypoxic exposure indicating a cell line and treatmentspecific metabolic adaptation. In cell line specific comparative volatilomic analysis, total 15 VOMs were found to be differentially abundant in aggressive cells with respect to non-aggressive cells in hypoxic condition and $12 \mathrm{VOMs}$ in normoxic conditions respectively. Out of these $27 \mathrm{VOMs}$, nine VOMs were found to be uniquely associated with the hypoxic condition whereas six VOMs were specific for the normoxic condition and six VOMs were observed to be common for both the conditions. Formic acid was identified as 
the most prominently abundant VOM in aggressive cells under both hypoxia and normoxia, indicating an existence of formate overflow in aggressive cells as compared to nonaggressive BC cells. Moreover, medium chain fatty acids such as n-decanoic acid and octanoic acid were observed at reduced levels in aggressive cells under normoxic condition specifying their higher consumption for fatty acid biosynthesis. While, ketonic metabolites such as 4-methyl-3penten-2-one, and 3-hexen-2-one show reduced abundance in aggressive cells as compared to non-aggressive cells, which indicate their higher rate of utilization under hypoxic condition. These data suggest that under hypoxic condition, aggressive cells prefer to be energetically efficient while under normoxic condition fatty acid biosynthesis is favoured
Fig. 4 Comparative volatilomic analysis of aggressive and nonaggressive cells grown in either hypoxic or normixic condition. a Hypoxia associated unique VOMs identified in aggressive vs. nonaggressive comparison. b Normoxia associated unique VOMs identified in aggressive vs. non-aggressive comparison. c VOMs common in both hypoxic as well as normoxic condition in aggressive vs. nonaggressive comparison. Green colour of box plot represents aggressive and red colour box plot represents non-aggressive BC cell line

as compared to non-aggressive cells indicating preference towards cell proliferation. This study further paves way for more mechanistic studies to understand the role of identified VOMs in the cancer aggression under hypoxic condition. a

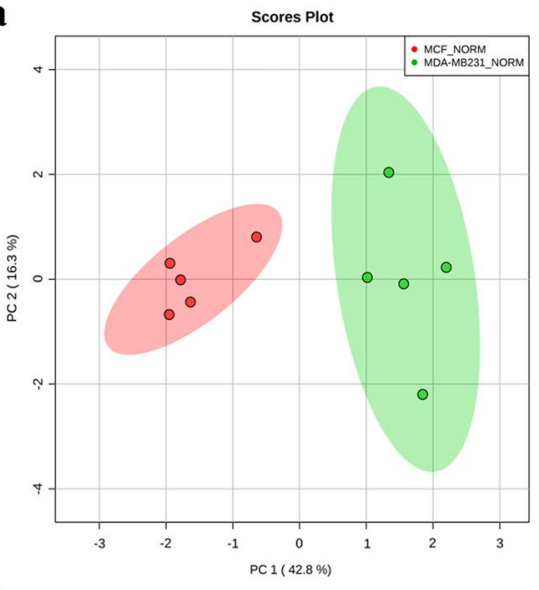

b

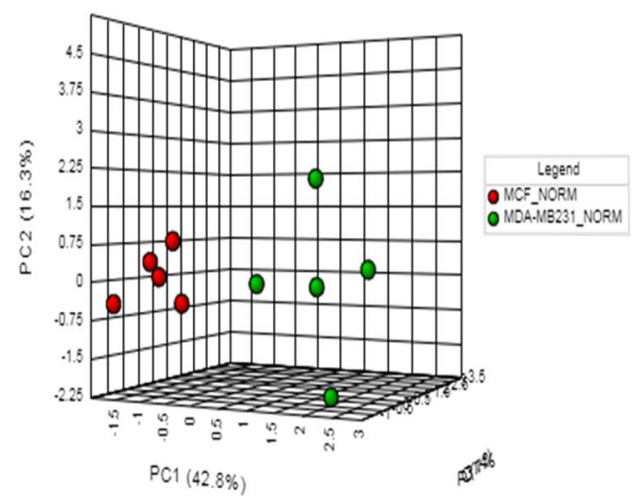

c

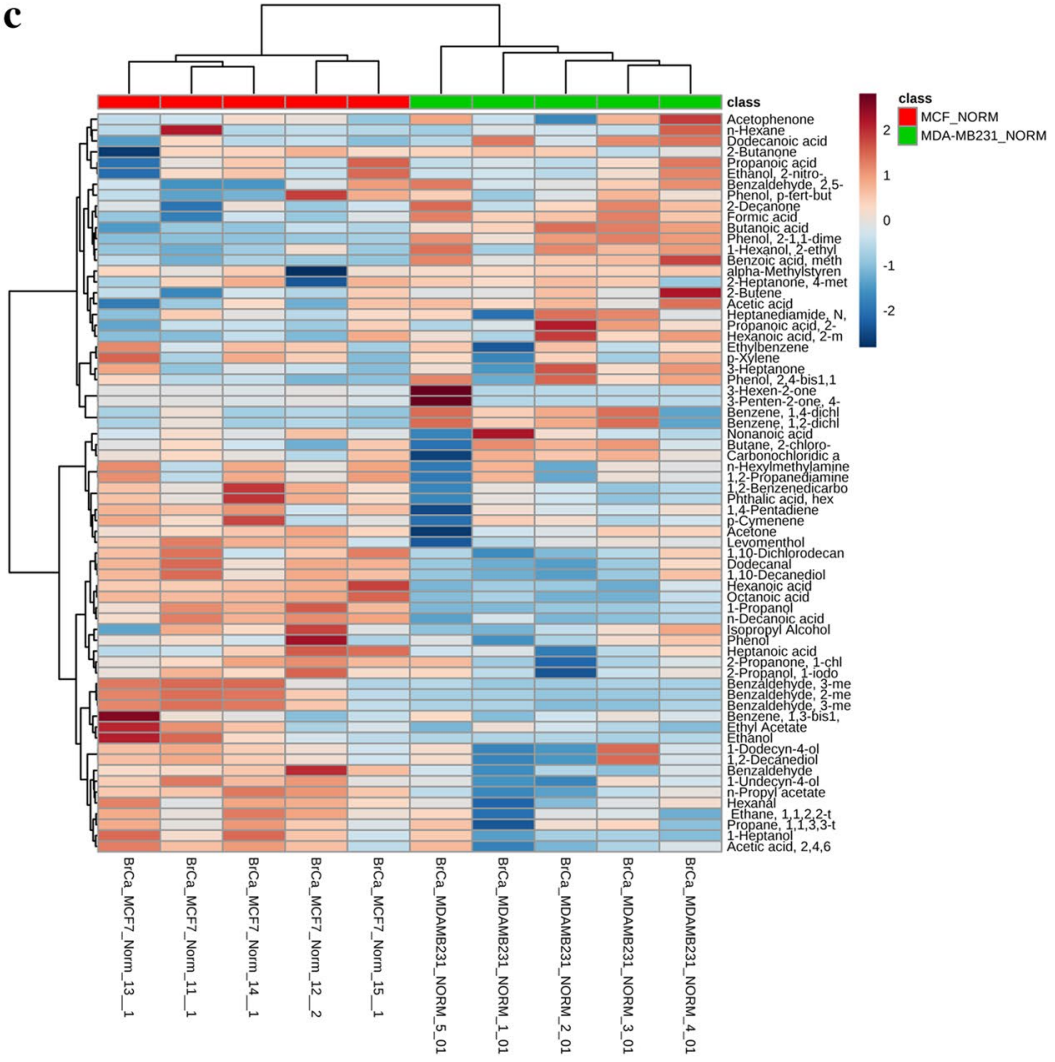

Fig. 3 a PCA 2D score plot and b PCA 3D score plot of the VOMs secreted by non-aggressive and aggressive cells under normoxic condition. c Hierarchical cluster analysis (HCA) of the VOMS secreted by non-aggressive and aggressive cells grown under normoxic condition represented as heatmap. Euclidean algorithm was used for the clustering and distance was measured by Ward Distance calculations 

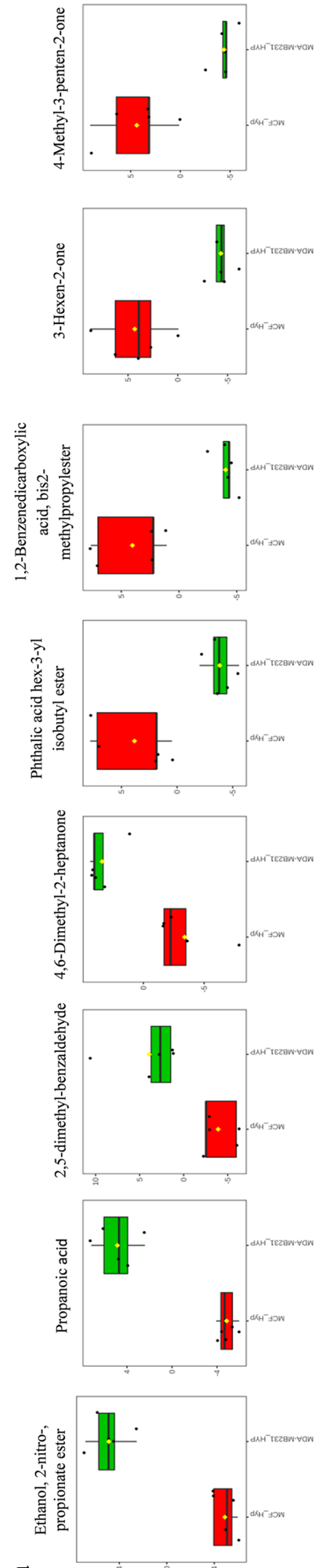
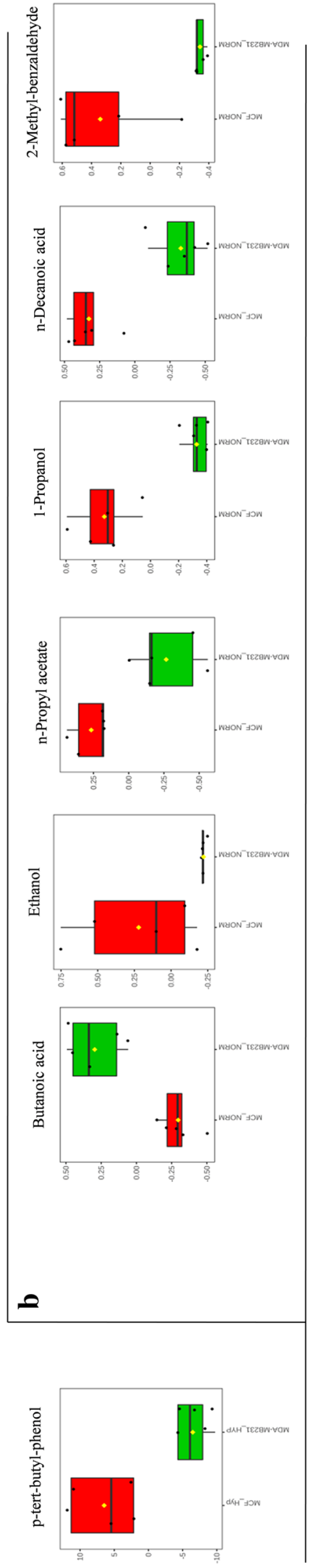
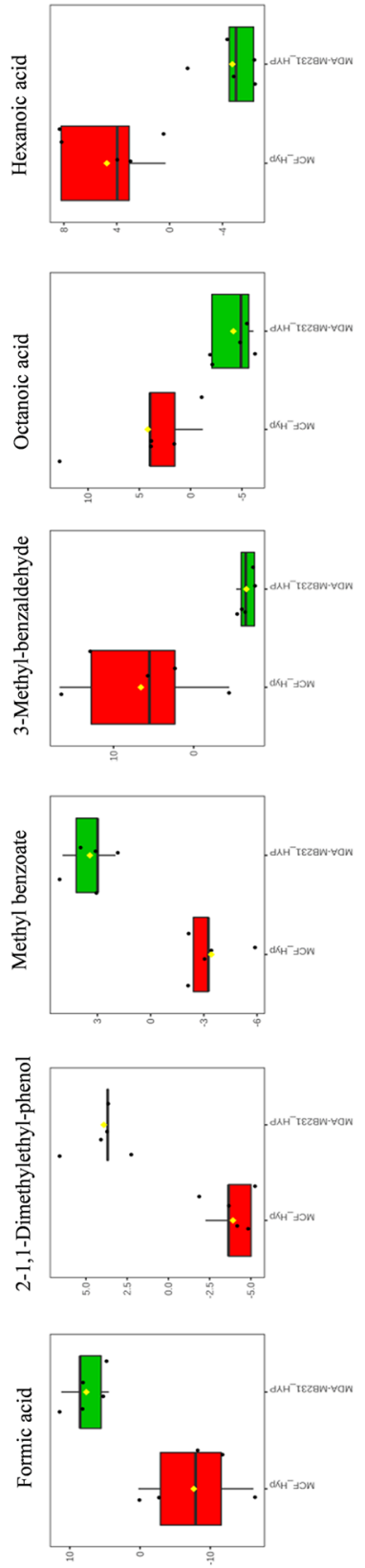

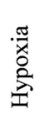
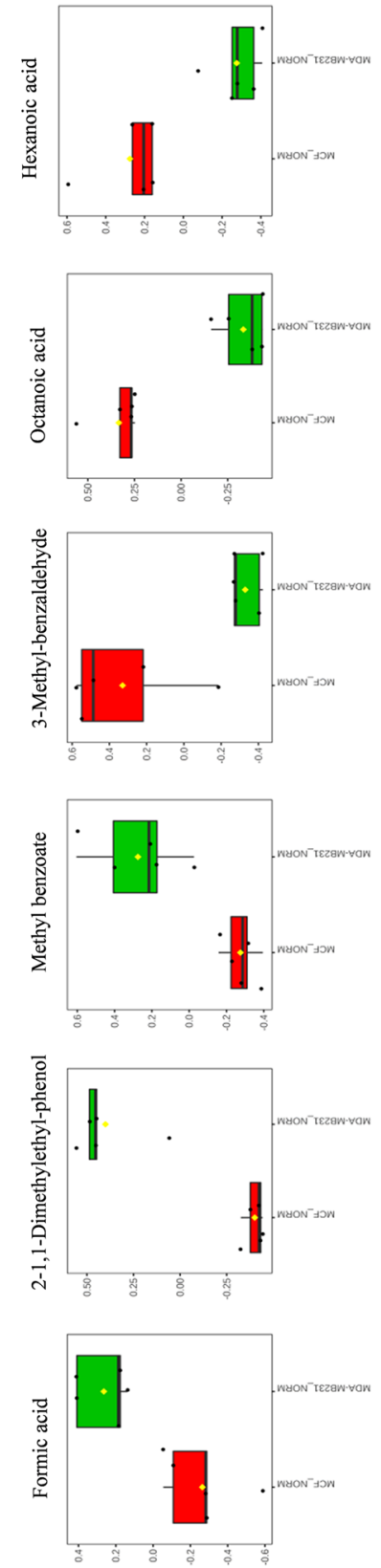

$\frac{\pi}{4}$
$\vdots$
$\vdots$
$z$ 

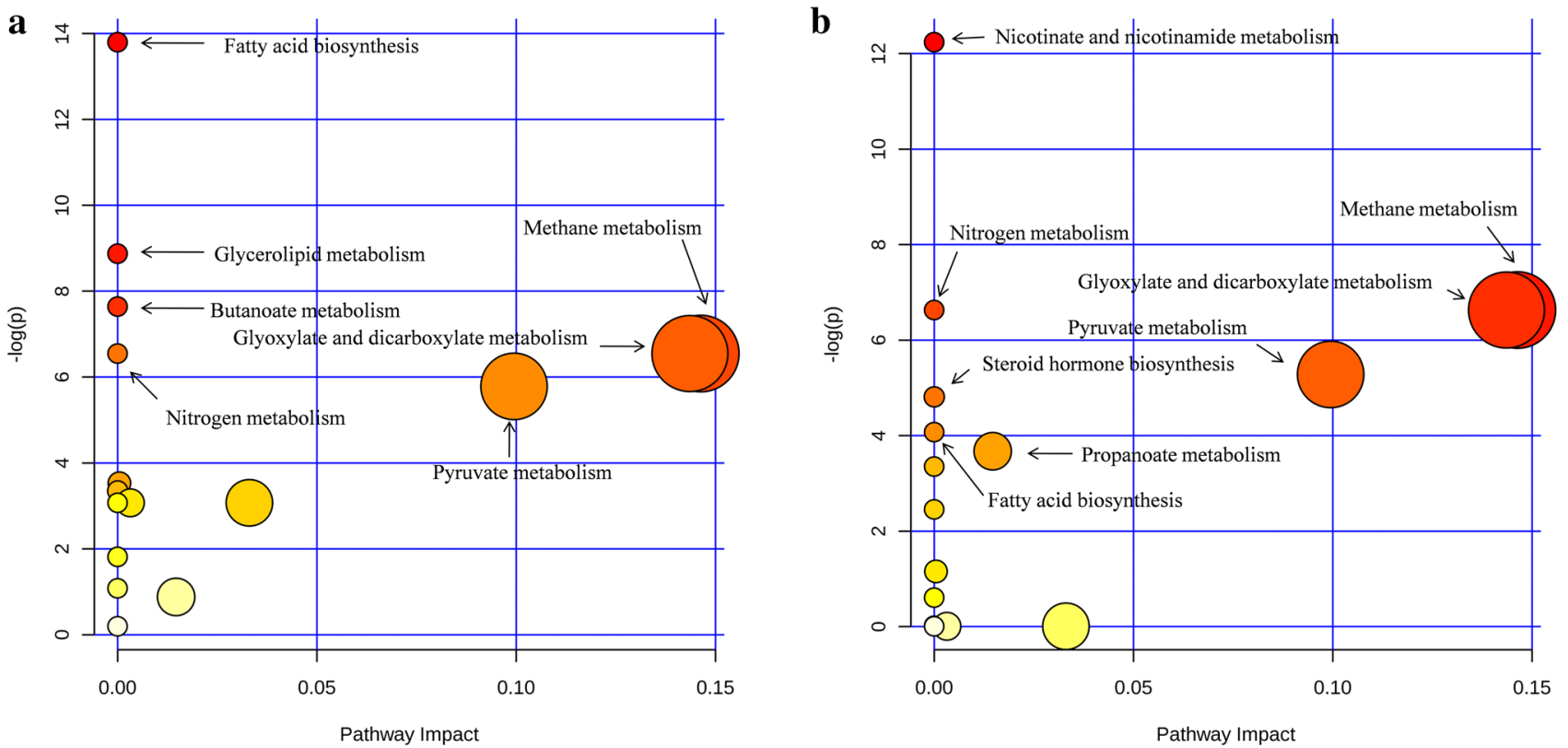

Fig. 5 Metabolic pathway alterations observed in the aggressive cells vs. non aggressive cells under a normoxia and $\mathbf{b}$ hypoxia

Acknowledgements This research was supported by Inno-Indigo NCD-CAPomics project, Department of Science \& Technology, Govt. of India, grant no. DST/IMRCD/EU/Inno-Indigo/NCDsCAPomics/2015. RT acknowledges Council of Scientific and Industrial Research, New Delhi, India for research associateship.This work also supported by FCT - Fundação para a Ciência e Tecnologia (project PEst-UID/QUI/UI0674/2019, CQM, New-INDIGO/0003/2012 and INNOINDIGO/0001/2015, Portuguese Government funds), Madeira 14-20 Program (project PROEQUIPRAM - Reforço do Investimentoem Equipamentos e Infraestruturas Científicasna RAM - M142001-0145-FEDER-000008) and ARDITI - Agência Regional para o Desenvolvimento da Investigação Tecnologia e Inovação (projects M1420-01-0145-FEDER-000005 - Centro de Química da Madeira CQM+(Madeira 14-20), and M1420-09-5369-FSE-000001) for the financial support and the post-doctoral fellowship granted to Jorge A. M. Pereira.

Authors Contributions Conceived the study: RT, KT, SR; Designed the study: RT, KT, TVSK, GCK, SR; Performed the experiments: RT, KT, TVSK; Compiled and analysed data: RT, KT, TVSK, JAMP, JSC, HAN, GCK, SR; Statistical analysis: RT, KT, HAN, SR; Provided instrument, chemicals and reagents: SR. All authors reviewed the manuscript and contributed in writing.

\section{Compliance with ethical standards}

Conflict of interest There are no conflicts to declare.

\section{References}

Al-Lahham, S. H., Peppelenbosch, M. P., Roelofsen, H., Vonk, R. J., \& Venema, K. (2010). Biological effects of propionic acid in humans; metabolism, potential applications and underlying mechanisms. Biochimica et Biophysica Acta-Molecular and Cell
Biology of Lipids,1801(11), 1175-1183. https://doi.org/10.1016/j. bbalip.2010.07.007.

Armitage, E. G., Kotze, H. L., Allwood, J. W., Dunn, W. B., Goodacre, R., \& Williams, K. J. (2015). Metabolic profiling reveals potential metabolic markers associated with Hypoxia Inducible Factormediated signalling in hypoxic cancer cells. Scientific Reports,5, 15649. https://doi.org/10.1038/srep15649.

Bray, F., Ferlay, J., Soerjomataram, I., Siegel, R. L., Torre, L. A., \& Jemal, A. (2018). Global cancer statistics 2018: GLOBOCAN estimates of incidence and mortality worldwide for 36 cancers in 185 countries. CA: A Cancer Journal for Clinicians, 68(6), 394-424. https://doi.org/10.3322/caac.21492.

Cavaco, C., Pereira, J. A. M., Taunk, K., Taware, R., Rapole, S., Nagarajaram, H., et al. (2018). Screening of salivary volatiles for putative breast cancer discrimination: An exploratory study involving geographically distant populations. Analytical and Bioanalytical Chemistry,410(18), 4459-4468. https://doi.org/10.1007/s0021 6-018-1103-x.

Chaturvedi, P., Gilkes, D. M., Wong, C. C. L., Luo, W., Zhang, H., Wei, H., et al. (2013). Hypoxia-inducible factor-dependent breast cancer-mesenchymal stem cell bidirectional signaling promotes metastasis. Journal of Clinical Investigation,123(1), 189-205. https://doi.org/10.1172/JCI64993.

Chen, Z., Li, Y., Zhang, H., Huang, P., \& Luthra, R. (2010). Hypoxiaregulated microRNA-210 modulates mitochondrial function and decreases ISCU and COX10 expression. Oncogene,29(30), 4362-4368. https://doi.org/10.1038/onc.2010.193.

Denko, N. C., Fontana, L. A., Hudson, K. M., Sutphin, P. D., Raychaudhuri, S., Altman, R., et al. (2003). Investigating hypoxic tumor physiology through gene expression patterns. Oncogene,22(37 REV. ISS. 3), 5907-5914. https://doi.org/10.1038/sj.onc.1206703.

Fasanaro, P., Greco, S., Lorenzi, M., Pescatori, M., Brioschi, M., Kulshreshtha, R., et al. (2009). An integrated approach for experimental target identification of hypoxia-induced miR-210. Journal of Biological Chemistry,284(50), 35134-35143. https://doi. org/10.1074/jbc.M109.052779. 
Favaro, E., Lord, S., Harris, A. L., \& Buffa, F. M. (2011). Gene expression and hypoxia in breast cancer. Genome Medicine,3(8), 55. https://doi.org/10.1186/gm271.

Gao, X., Lin, S. H., Ren, F., Li, J. T., Chen, J. J., Yao, C. B., et al. (2016). Acetate functions as an epigenetic metabolite to promote lipid synthesis under hypoxia. Nature Communications, 7, 11960. https://doi.org/10.1038/ncomms11960.

Griffin, J. L., Nicholls, A. W., Daykin, C. A., Heald, S., Keun, H. C., Schuppe-Koistinen, I., et al. (2007). Standard reporting requirements for biological samples in metabolomics experiments: Mammalian/in vivo experiments. Metabolomics,3(3), 179-188. https:// doi.org/10.1007/s11306-007-0077-z.

Harris, A. L. (2002). Hypoxia-A key regulatory factor in tumour growth. Nature Reviews Cancer,2(1), 38-47. https://doi. org/10.1038/nrc704.

Huang, X., Ding, L., Bennewith, K. L., Tong, R. T., Welford, S. M., Ang, K. K., et al. (2009). Hypoxia-inducible mir-210 regulates normoxic gene expression involved in tumor initiation. Molecular Cell,35(6), 856-867. https://doi.org/10.1016/j.molce 1.2009.09.006.

Intlekofer, A. M., DeMatteo, R. G., Venneti, S., Finley, L. W. S., Lu, C., Judkins, A. R., et al. (2015). Hypoxia induces production of L-2-hydroxyglutarate. Cell Metabolism,22(2), 304-311. https:// doi.org/10.1016/j.cmet.2015.06.023.

Jaakkola, P., Mole, D. R., Tian, Y. M., Wilson, M. I., Gielbert, J., Gaskell, S. J., et al. (2001). Targeting of HIF- $\alpha$ to the von HippelLindau ubiquitylation complex by $\mathrm{O}_{2}$-regulated prolyl hydroxylation. Science,292(5516), 468-472. https://doi.org/10.1126/scien ce. 1059796.

Martinez-Outschoorn, U. E., Lin, Z., Whitaker-Menezes, D., Howell, A., Lisanti, M. P., \& Sotgia, F. (2012). Ketone bodies and two-compartment tumor metabolism: Stromal ketone production fuels mitochondrial biogenesis in epithelial cancer cells. Cell Cycle,11(21), 3956-3963.

Mashimo, T., Pichumani, K., Vemireddy, V., Hatanpaa, K. J., Singh, D. K., Sirasanagandla, S., et al. (2014). Acetate is a bioenergetic substrate for human glioblastoma and brain metastases. Cell,159(7), 1603-1614. https://doi.org/10.1016/j.cell.2014.11.025.

Meiser, J., Schuster, A., Pietzke, M., Voorde, J. V., Athineos, D., Oizel, K., et al. (2018). Increased formate overflow is a hallmark of oxidative cancer. Nature Communications,9(1), 1368. https://doi. org/10.1038/s41467-018-03777-w.

Metallo, C. M., Gameiro, P. A., Bell, E. L., Mattaini, K. R., Yang, J., Hiller, K., et al. (2012). Reductive glutamine metabolism by IDH1 mediates lipogenesis under hypoxia. Nature,481(7381), 380-384. https://doi.org/10.1038/nature10602.

O'Shaughnessy, J. (2005). Extending survival with chemotherapy in metastatic breast cancer. The Oncologist,10(suppl_3), 20-29. https ://doi.org/10.1634/theoncologist.10-90003-20.

Prabhakar, N. R., \& Semenza, G. L. (2012). Adaptive and maladaptive cardiorespiratory responses to continuous and intermittent hypoxia mediated by hypoxia-inducible factors 1 and 2. Physiological Reviews,92(3), 967-1003. https://doi.org/10.1152/physr ev.00030.2011.

Semenza, G. L. (2010a). Defining the role of hypoxia-inducible factor 1 in cancer biology and therapeutics. Oncogene,29(5), 625-634. https://doi.org/10.1038/onc.2009.441.

Semenza, G. L. (2010b). HIF-1: upstream and downstream of cancer metabolism. Current Opinion in Genetics and Development,20(1), 51-56. https://doi.org/10.1016/j.gde.2009.10.009.

Semenza, G. L. (2012). Hypoxia-inducible factors in physiology and medicine. Cell,148(3), 399-408. https://doi.org/10.1016/j. cell.2012.01.021.

Semenza, G. L. (2016). The hypoxic tumor microenvironment: A driving force for breast cancer progression. Biochimica et Biophysica
Acta-Molecular Cell Research,1863(3), 382-391. https://doi. org/10.1016/j.bbamcr.2015.05.036.

Silva, C. L., Passos, M., \& Câmara, J. S. (2011). Investigation of urinary volatile organic metabolites as potential cancer biomarkers by solid-phase microextraction in combination with gas chromatography-mass spectrometry. British Journal of Cancer,105(12), 1894-1904.

Taunk, K., Taware, R., More, T. H., Porto-figueira, P., Pereira, J. A. M., Mohapatra, R., et al. (2018). A non-invasive approach to explore the discriminatory potential of the urinary volatilome of invasive ductal carcinoma of the breast. RSC Advances, 8(44), 25040-25050. https://doi.org/10.1039/C8RA02083C.

Taware, R., Taunk, K., Pereira, J. A. M., Dhakne, R., Kannan, N., Soneji, D., et al. (2017). Investigation of urinary volatomic alterations in head and neck cancer: A non-invasive approach towards diagnosis and prognosis. Metabolomics,13(10), 111. https://doi. org/10.1007/s11306-017-1251-6.

Taware, R., Taunk, K., Pereira, J. A. M., Shirolkar, A., Soneji, D., Câmara, J. S., et al. (2018). Volatilomic insight of head and neck cancer via the effects observed on saliva metabolites. Scientific Reports,8(1), 17725. https://doi.org/10.1038/s41598-018-35854 -x.

Tsai, I. L., Kuo, T. C., Ho, T. J., Harn, Y. C., Wang, S. Y., Fu, W. M., et al. (2013). Metabolomic dynamic analysis of hypoxia in MDAMB-231 and the comparison with inferred metabolites from transcriptomics data. Cancers,5(2), 491-510. https://doi.org/10.3390/ cancers5020491.

Vaupel, P. (2004). Tumor hypoxia: Causative factors, compensatory mechanisms, and cellular response. The Oncologist,9(suppl_5), 4-9. https://doi.org/10.1634/theoncologist.9-90005-4.

Vaupel, P., Höckel, M., \& Mayer, A. (2007). Detection and characterization of tumor hypoxia using $\mathrm{pO}_{2}$ histography. Antioxidants \& Redox Signaling,9(8), 1221-1236. https://doi.org/10.1089/ ars.2007.1628.

Wang, G. L., Jiang, B. H., Rue, E. A., \& Semenza, G. L. (1995). Hypoxia-inducible factor 1 is a basic-helix-loop-helix-PAS heterodimer regulated by cellular $\mathrm{O}_{2}$ tension. Proceedings of the National Academy of Sciences of the United States of America,92(12), 5510-5514

Warburg, O. (1956). Injuring of respiration the origin of cancer cells. Science,123, 309-314. https://doi.org/10.1126/scien ce.123.3191.309.

Warburg, O., Wind, F., \& Negelein, E. (1927). THE Metabolism of tumors in the body. The Journal of General Physiology,8(6), 519-530. https://doi.org/10.1085/jgp.8.6.519.

Weidemann, A., \& Johnson, R. S. (2008). Biology of HIF-1 $\alpha$. Cell Death and Differentiation,15(4), 621-627. https://doi. org/10.1038/cdd.2008.12.

Xia, J., \& Wishart, D. S. (2011). Web-based inference of biological patterns, functions and pathways from metabolomic data using MetaboAnalyst. Nature Protocols,6(6), 743-760. https://doi. org/10.1038/nprot.2011.319.

Xia, J., Psychogios, N., Young, N., \& Wishart, D. S. (2009). MetaboAnalyst: A web server for metabolomic data analysis and interpretation. Nucleic Acids Research,37(SUPPL. 2), 652-660. https ://doi.org/10.1093/nar/gkp356.

Xia, J., Wishart, D. S., \& Valencia, A. (2011). MetPA: A web-based metabolomics tool for pathway analysis and visualization. Bioinformatics, 27, 2342-2344. https://doi.org/10.1093/bioinforma tics/btq418.

Yang, J., Cheng, J., Sun, B., Li, H., Wu, S., Dong, F., et al. (2018). Untargeted and stable isotope-assisted metabolomic analysis of MDA-MB-231 cells under hypoxia. Metabolomics, 14(4), 40. https ://doi.org/10.1007/s11306-018-1338-8.

Zhang, C., Samanta, D., Lu, H., Bullen, J. W., Zhang, H., Chen, I., et al. (2016). Hypoxia induces the breast cancer stem cell phenotype by 
HIF-dependent and ALKBH5-mediated m 6 A-demethylation of NANOG mRNA. Proceedings of the National Academy of Sciences of the United States of America, 113(14), E2047-E2056. https://doi.org/10.1073/pnas.1602883113.
Publisher's Note Springer Nature remains neutral with regard to jurisdictional claims in published maps and institutional affiliations. 